\title{
Scolia
}

Revue de linguistique

\section{Les sujets disloqués et non-disloqués chez l'enfant français}

Dislocated and Non-Dislocated Subjects in Child French

Morgane Jourdain et Karen Lahousse

\section{CpenEdition}

Édition électronique

URL : http://journals.openedition.org/scolia/1073

DOI : $10.4000 /$ scolia. 1073

ISSN : 2677-4224

Éditeur

Presses universitaires de Strasbourg

Édition imprimée

Date de publication : 10 juillet 2020

Pagination : 13-31

ISBN : 979-10-344-0067-6

ISSN : 1253-9708

Référence électronique

Morgane Jourdain et Karen Lahousse, «Les sujets disloqués et non-disloqués chez l'enfant français », Scolia [En ligne], 34 | 2020, mis en ligne le 10 juillet 2020, consulté le 10 juillet 2020. URL : http://

journals.openedition.org/scolia/1073; DOI : https://doi.org/10.4000/scolia.1073 
SCOLIA 34/2020, p. 13-31.

\title{
Les sujets disloqués et non-disloqués chez l'enfant français
}

\author{
Morgane JouRDAIN \\ KU Leuven, Université de Lille, laboratoire STL \\ Morgane.jourdain@kuleuven.be \\ Karen LAHOUSSE \\ KU Leuven \\ Karen.lahousse@kuleuven.be
}

\section{Introduction}

Le but de cet article est d'identifier chez l'enfant français les différences entre les sujets disloqués (1) et non-disloqués (2) au niveau de la structure de l'information (SI).

1) Fanfan il a eu le cadeau comme ça. (Héloïse, 2 ans, corpus TCOF, ATILF, 2018)

2) Une fois, une vieille sonna à la porte. (Adeline, 5 ans, corpus TCOF, ATILF, 2018)

Nous avons extrait 370 énoncés avec sujet disloqué (SD), et 1029 avec un sujet non-disloqué (SND), produits par 21 enfants du corpus TCOF (ATILF, 2018). Les propriétés de la SI des pronoms et des expressions lexicales étant différentes (Prince, 1981), nous présenterons deux analyses, une concernant les sujets pronominaux disloqués et non-disloqués, et une autre pour les sujets lexicaux disloqués et nondisloqués. Nous montrerons que: (i) comme chez l'adulte, les SD sont généralement moins accessibles pour l'interlocuteur que les SND; (ii) les enfants produisent des topiques contrastifs avec des SD mais pas 
avec des SND; (iii) les enfants produisent des SD uniquement dans des énoncés avec configuration topique-commentaire, alors que les SND peuvent faire partie du focus.

Nous mettrons ces résultats en regard avec les études de Gotowski (2015) et Legendre, Culbertson, Barrière, Nazzi, \& Goyet (2010), qui considèrent que les enfants interprètent le pronom clitique comme étant un marqueur morphologique du verbe, et donc comme étant obligatoire. Selon ces auteurs, cette hypothèse explique pourquoi les enfants produisent presque systématiquement des SD si le sujet est lexical. Nous montrerons que compte tenu des divergences de propriétés de la SI entre les SD et SND chez l'enfant, nos résultats ne semblent pas soutenir cette hypothèse, et semblent plutôt indiquer, comme suggéré par De Cat $(2007,2009)$ que les enfants expriment le sujet sous la forme requise selon ses propriétés informationnelles. Le faible nombre de SND lexicaux ne nous permet cependant pas de rejeter avec certitude l'hypothèse du traitement du pronom clitique comme marqueur morphologique.

L'article est structuré de la façon suivante: dans la section 1, nous donnerons un aperçu de la littérature sur la dislocation en français, chez l'adulte et chez l'enfant. Puis, dans la section 2, nous expliquerons comment nous avons extrait nos données, et comment nous avons analysé leurs propriétés au niveau de la SI. Dans la section 3, nous comparerons les SD et SND pronominaux, puis les SD et SND lexicaux. Dans la section 4, nous confronterons nos résultats aux hypothèses de Gotowski (2015) et Legendre et al. (2010).

\section{1. État de la question}

\subsection{La dislocation en français chez l'adulte}

La dislocation est définie comme la production d'un constituant en périphérie gauche ou droite de la phrase, qui est repris par un pronom clitique à l'intérieur de la proposition (Delais-Roussarie et al., 2004; Lambrecht, 2001)1. Il existe d'autres types de constituants

$1 \quad$ Il est courant dans la littérature de différencier les constituants disloqués marqués par le même cas que le pronom clitique (appelés Clitic Dislocation) de ceux qui ne le sont pas (appelés Hanging Topics) (Cinque, 1983; Delais-Roussarie, Doetjes, \& Sleeman, 2004). Comme cet article concerne uniquement le sujet, et qu'il n'est 
disloqués ne nécessitant pas de reprise par un pronom clitique (voir Abeillé, Godard, \& Sabio, 2008 et Lagae, 2012 pour une présentation des différents types de constituants détachés en français), mais dans la présente étude, nous nous concentrons sur les dislocations avec reprise.

Cette construction a une articulation topique-commentaire (Campion, 1984; De Cat, 2007; Lambrecht, 2001), c'est-à-dire que l'élément disloqué doit correspondre à un référent appartenant aux connaissances partagées entre les interlocuteurs, et le reste de la phrase apporte une information nouvelle au sujet de ce référent (Lambrecht, 1994; Reinhart, 1981).

3) A: Quand on y était allés, on était euh logés dans un loft euh à Manhattan. La nénette, ah c'était génial, quoi, l'usine désaffectée, enfin qui ét/ qui était plus désaffectée quoi, qui avait été euh $r$ / rénovée, remise en loft, elle avait un loft immense qui donnait sur les deux grandes tours là. Et pui c'était euh, splendide quoi. B: Elle s'appelait Loana, la nénette? (Horváth, 2016: 253)

Dans la dislocation présentée en (3), le constituant la nénette se rapporte à un référent appartenant aux connaissances partagées entre les interlocuteurs, puisqu'il est introduit dans le contexte gauche. Le commentaire, elle s'appelait Loana, exprime, ou dans ce contexte-ci, demande, une information nouvelle au sujet du topique la nénette.

\subsection{La dislocation chez l'enfant}

La dislocation est plus fréquente chez l'enfant que chez l'adulte (Parisse, 2008). Plusieurs études mettent en doute le fait que la dislocation chez l'enfant obéisse aux mêmes règles syntaxiques que chez l'adulte. Selon Legendre et al. (2010) et Gotowski (2015), les enfants interprètent le pronom clitique comme étant un marqueur morphologique flexionnel rattaché au verbe ${ }^{2}$. Ainsi, tout sujet lexical, quel que soit son statut syntaxique, ou son statut informationnel serait disloqué, ce qui expliquerait la plus grande fréquence de sujets disloqués

pas possible de déterminer si un constituant disloqué sujet est marqué par un cas (Blasco-Dulbecco, 1999), nous ne ferons pas de distinction entre les dislocations clitiques et les hanging topics.

2 Selon certains auteurs, le pronom clitique aurait le statut de marqueur flexionnel chez l'adulte également (Auger, 1994; Culbertson, 2010 mais voir De Cat, 2005 pour une conclusion opposée). 
chez l'enfant que chez l'adulte. L'un des principaux arguments en faveur de cette interprétation est la production de dislocations avec des NPs avec quantificateurs (4a.) ou des NPs indéfinis à sens non général (4b.) (Ferdinand, 1996; Legendre et al., 2010).

4) a. Tout le monde il veut une cigarette. (Philippe, $2 ; 2$ )

b. Une voiture elle roule. (Philippe, 2;3) (Ferdinand, 1996)

Ces dislocations ne sont pas typiques du discours de l'adulte (Barnes, 1985; De Cat, 2007; Horváth, 2018), puisque ces types de syntagmes ne peuvent pas être le topique (Lambrecht, 1994). Les énoncés en (4) suggèrent donc que les enfants traitent le pronom clitique sujet comme obligatoire, ce qui peut s'expliquer s'il est interprété comme marqueur morphologique du verbe. Ainsi, tous les sujets lexicaux, même ceux ne correspondant pas au topique de la phrase, seraient disloqués.

Parisse (2008), quant à lui, émet l'hypothèse que la dislocation est utilisée par l'enfant pour diminuer la charge cognitive des énoncés ayant un sujet lexical. En disloquant le sujet, l'énoncé est séparé en deux parties (5), ce qui en faciliterait la production.

5) Oui mais ma petite soeur, elle a déjà une lampe. (Gabriel, 4 ans, corpus TCOF)

Cependant, en ce qui concerne l'aspect informationnel de la construction, les recherches expérimentales et les analyses de corpus indiquent que les enfants utilisent la dislocation avec une articulation topique-commentaire, comme chez l'adulte, dès le début de son acquisition (Belletti \& Manetti, 2018; De Cat, 2007, 2009). Plus précisément, De Cat (2009) montre que dès l'âge de 2,6 ans au moins, les enfants produisent les différents types de sujets disponibles en français (sujets lexicaux, pronoms, disloqués, clivés, etc.) dans des contextes similaires à ceux des adultes.

\subsection{But de notre étude}

Si les enfants utilisent la dislocation dans les contextes appropriés, cela pourrait suggérer qu'ils ne traitent pas le pronom clitique comme marqueur morphologique du verbe, puisque sa présence dépendrait du contexte. Cela montrerait également que l'hypothèse de la dislocation comme moyen de séparer l'énoncé en deux pour en faciliter sa production (Parisse, 2008) ne serait pas la plus appropriée pour rendre compte des productions de l'enfant. Les études en faveur d'une 
interprétation morphologique du pronom clitique ou d'un allègement cognitif ne considèrent pas le contexte de production des SD et SND, bien qu'il soit déterminant dans le choix du type de sujet chez l'adulte (Lambrecht, 1987). Le but de notre recherche est donc d'identifier les contextes entraînant la production de SD ou SND chez l'enfant français, pour déterminer si le pronom clitique a le statut d'argument ou de marqueur morphologique du verbe.

Dans notre analyse, nous séparerons les résultats pour les sujets pronominaux (SP) et sujets lexicaux (SL), car les pronoms et syntagmes lexicaux ont des propriétés différentes au niveau de la SI (Prince, 1981). Pour les SL, nous regarderons si le sujet correspond au topique, ou s'il appartient au focus de l'énoncé. De Cat (2002; 2007), s'appuyant sur Lambrecht (1994), montre en effet que lorsque le sujet lexical est topique, il doit être obligatoirement disloqué, comme illustré en (6). Ainsi, selon ces auteurs, en français, les SL non-disloqués (SLND) appartiennent obligatoirement au focus de la phrase, tandis que les SL disloqués (SLD) correspondent toujours au topique.

6) a. Le malais, c'est difficile.

b. \#Le malais est difficile. (De Cat, 2002)

Pour les SP, nous considérerons dans un premier tempsl'accessibilité du référent. Les SPND correspondent en général au degré maximal d'accessibilité (Prince, 1981), ce qui n'est pas obligatoirement le cas des sujets disloqués (Ashby, 1988; Barnes, 1985; De Cat, 2007; Horváth, 2018; Lambrecht, 2001). Ensuite, nous analyserons le statut contrastif du sujet, car la dislocation est fréquemment utilisée pour exprimer un topique contrastif (Ashby, 1988; Horváth, 2018).

\section{Méthodologie}

\subsection{Participants}

Nous avons effectué une analyse de conversations adulte-enfant, pour un total de 21 enfants entre 2 et 5 ans du corpus TCOF (ATILF, 2018). Nous avons établi des tranches d'âge de 6 mois, et avons extrait les dislocations des enregistrements de trois enfants par tranche d'âge.

Les interactions sont spontanées, et ont eu lieu au domicile de l'enfant. L'adulte interagissant avec l'enfant est généralement un étudiant en linguistique. 


\subsection{Classification en SD et SND}

Nous avons extrait tous les énoncés produits par les enfants de notre corpus, et les avons classés entre SD et SND. Durant notre classification, nous avons exclu les énoncés à l'impératif, ou ceux dont le sujet est impersonnel. Nous avons également exclu les sujets des propositions subordonnées relatives ou les sujets clivés.

Nous avons utilisé la présence du pronom clitique comme critère de répartition entre $\mathrm{SD}$ et $\mathrm{SND}$. Les pronoms clitiques sujets sont en effet acquis avant 2 ans (Hamann, Rizzi, \& Frauenfelder, 1996; Legendre, Vainikka, \& Todorova, 2002; Pierce, 1992), et constituent donc un critère solide pour séparer les SD des SND. Cependant, chez les enfants les plus jeunes, le pronom clitique peut tout de même être manquant dans les dislocations. Ces cas se divisent en deux catégories dans nos données (i) les sujets produits en fin d'énoncé (7), que nous avons classés comme dislocation suivant Labelle \& Valois (1996) et Ferdinand (1996; 1997), et (ii) les pronoms sujets toniques (8), extraits en tant que sujets pronoms disloqués (SPD), suivant De Cat (2004; 2007).

7) Parce que encore petit lui. (Charlotte, 2 ans)

8) Moi fais du découpage maintenant. (Fantine, 2,5 ans)

\subsection{Codage des propriétés de la SI}

\subsubsection{La configuration de la SI}

Nous basant sur les définitions du topique de Lambrecht (1994) et Reinhart (1981), nous interprétons le sujet comme étant le topique de l'énoncé s'il appartient aux connaissances partagées entre les interlocuteurs, et si le reste de la phrase apporte une information nouvelle, non-mentionnée dans le discours précédent, au sujet de ce référent, comme illustré en (9). Dans cet exemple, l'élément disloqué est les fleurs. Il appartient aux connaissances partagées entre les interlocuteurs, puisqu'il est réalisé dans le contexte gauche. L'énoncé de l'enfant donne une information nouvelle qui doit être rattachée à ce référent. Les fleurs fonctionne donc comme le topique de l'énoncé.

9) Adulte: Pourquoi t'aimes pas les fleurs? Enfant: Parce que c'est pas gentil, les fleurs. (Charlotte, 2 ans) 
Il est possible d'utiliser un test de topicalité, pour vérifier le statut d'un référent (Erteschik-Shir, 2007; Reinhart, 1981). Nous illustrons ce test en (10), qui est une transformation de l'énoncé (9).

10) - Et à propos des fleurs? / Parle-moi des fleurs.

- Je dis à propos des fleurs qu'elles ne sont pas gentilles.

Nous considérons qu'un sujet appartient au focus de la phrase s'il apporte une information différente de ce qui est présupposé par le contexte précédent (Lambrecht, 1994). Par exemple, en (11), l'enfant commence à raconter une histoire. L'ensemble de l'énoncé constitue un événement correspondant à une information nouvelle, différente du contexte gauche. Le référent une vieille n'est jamais mentionné avant cet énoncé dans l'enregistrement.

11) Une fois, une vieille sonna à la porte. (Adeline, 5 ans)

\subsubsection{L’accessibilité}

Nous avons basé notre codage de l'accessibilité sur les travaux de Prince (1981). La première catégorie est mentionné, sans compétiteur. Les référents produits dans le contexte précédent (12), ainsi que les participants à l'interaction (13) appartiennent à cette catégorie.

12) Maintenant, il faut trouver l'éléphant. Où est-ce qu'il est, l'éléphant? (Laurie, 4 ans)

13) Toi, tu joues à ça. (Antonin, 2,5 ans)

Il est également possible qu'un référent soit déjà produit dans le contexte précédent, mais qu'il y ait des compétiteurs, c'est-à-dire d'autres référents également mentionnés dans le discours. Par exemple, en (14), le référent le bébé est mentionné dans la proposition précédente par le référent Juliette (le nom du bébé), mais un autre référent est mentionné, papa. Il y a donc deux référents mentionnés et actifs, Papa et Juliette, lorsque le constituant le bébé est produit. Nous appelons cette deuxième catégorie mentionné, avec compétiteur.

14) Papa crie Juliette parce que le bébé il a fait caca. (Marianne, 2 ans) Un référent peut ne pas avoir été produit dans le contexte linguistique, mais être physiquement présent dans la situation d'énonciation (15).

15) ça, c’est quoi? (Héloïse, 2 ans) 
Un référent peut également être inférable, s'il est lié sémantiquement à un autre référent qui lui a été produit dans le contexte linguistique précédent (16).

16) Adulte: Et à l'école, tu fais du coloriage?

Enfant: La maîtresse elle veut pas. (Laureen, 4 ans)

Enfin, un référent peut être nouveau, soit ne pas avoir été produit dans le contexte linguistique précédent, ne pas être lié sémantiquement à autre référent et ne pas être présent dans la situation d'énonciation.

\subsubsection{Le contraste}

Un topique est contrastif dans deux cas: (i) s'il existe, dans le discours précédent ou dans la situation d'énonciation, une alternative pour le même type de prédicat (17) et (ii) si le topique dans l'énoncé constitue une réponse partielle à une question avec topique plus large (18) (Büring, 2016; Repp, 2010).

17) Moi je joue à ça et toi tu joues à ça. (Antonin, 2,5 ans)

18) Adulte: Ensuite qu'est-ce qu'ils font?

Enfant: Anaïs elle met sa culotte et Ludovic il prend des chaussettes. (Louise, 4 ans)

\section{Résultats}

\subsection{Répartition des types de sujets}

Les types de sujets produits par les enfants de notre corpus sont présentés dans le tableau 1, et sont organisés par tranche d'âge.

\begin{tabular}{|c|c|c|c|c|}
\hline Age & SPND & SPD & SLND & SLD \\
\hline 2 & 62 & 5 & 2 & 11 \\
\hline 2,5 & 73 & 38 & 0 & 6 \\
\hline 3 & 110 & 44 & 0 & 18 \\
\hline 3,5 & 213 & 53 & 5 & 20 \\
\hline 4 & 211 & 43 & 2 & 28 \\
\hline 4,5 & 164 & 24 & 0 & 20 \\
\hline 5 & 185 & 23 & 2 & 37 \\
\hline Total & 1018 & 230 & 11 & 140 \\
\hline
\end{tabular}

Tableau 1 : Fréquence absolue des types de sujets par tranche d'âge 
Dans le tableau 1, nous pouvons constater que le type de sujet le plus fréquent est le SPND (pronom clitique simple). En ce qui concerne les SL, la plupart (93\%) sont disloqués. Les SPND, SPD et SLD (à part 14 SPD dont le clitique est manquant, voir section 2.2) ont tous un pronom clitique sujet exprimé. Le pronom clitique est donc absent seulement dans ces 14 SPD et dans les SLND. Les enfants de notre corpus produisent donc seulement 25 sujets sans pronom clitique, ce qui représente seulement $1,8 \%$ de tous leurs sujets exprimés.

Dans cette section, nous allons à présent comparer les différents types de sujets produits par les enfants de notre corpus. Comme indiqué dans l'introduction, les pronoms et expressions lexicales ont en général des propriétés informationnelles différentes, puisque les pronoms sont généralement plus accessibles que les syntagmes nominaux (Prince, 1981). Nous allons donc comparer les sujets disloqués et non-disloqués pour les pronoms, puis pour les syntagmes nominaux.

\subsection{SPD et SPND}

\subsubsection{Accessibilité}

Les pronoms sont généralement utilisés pour exprimer des référents hautement accessibles. Cependant, différents types de pronoms, comme les pronoms clitiques ou les pronoms déictiques, peuvent avoir différents degrés d'accessibilité (Prince, 1981). Le degré d'accessibilité des SPD est présenté dans le tableau 2, et le degré d'accessibilité de SPND est présenté dans le tableau 3.

\begin{tabular}{|c|c|c|c|c|c|c|c|}
\hline Age & $\begin{array}{c}\text { Mentionné, } \\
\text { sans comp. }\end{array}$ & $\begin{array}{c}\text { Mentionné, } \\
\text { avec comp. }\end{array}$ & $\begin{array}{c}\text { Physiquement } \\
\text { présent }\end{array}$ & Inférable & Nouveau & Ambigu & Total \\
\hline 2 & 1 & 2 & 2 & 0 & 0 & 0 & 5 \\
\hline 2,5 & 28 & 2 & 7 & 0 & 0 & 1 & 38 \\
\hline 3 & 15 & 0 & 24 & 0 & 0 & 5 & 44 \\
\hline 3,5 & 33 & 4 & 12 & 2 & 2 & 0 & 53 \\
\hline 4 & 26 & 6 & 11 & 0 & 0 & 0 & 43 \\
\hline 4,5 & 20 & 0 & 3 & 0 & 0 & 1 & 24 \\
\hline 5 & 13 & 3 & 4 & 2 & 0 & 1 & 23 \\
\hline Total & 136 & 17 & 63 & 4 & 2 & 8 & 230 \\
5 & $59 \%$ & $6 \%$ & $27 \%$ & $2 \%$ & $1 \%$ & $3 \%$ & $100 \%$ \\
\hline
\end{tabular}

Tableau 2: Accessibilité des SPD par tranche d'âge 


\begin{tabular}{|c|c|c|c|c|c|c|c|}
\hline Age & $\begin{array}{l}\text { Mentionné, } \\
\text { sans comp. }\end{array}$ & $\begin{array}{l}\text { Mentionné, } \\
\text { avec comp. }\end{array}$ & $\begin{array}{l}\text { Physiquement } \\
\text { présent }\end{array}$ & Inférable & Nouveau & Ambigu & Total \\
\hline 2 & 51 & 7 & 3 & 0 & 0 & 1 & 62 \\
\hline 2,5 & 49 & 14 & 10 & 0 & 0 & 0 & 73 \\
\hline 3 & 87 & 18 & 4 & 0 & 0 & 1 & 110 \\
\hline 3,5 & 194 & 10 & 6 & 2 & 0 & 1 & 213 \\
\hline 4 & 194 & 10 & 5 & 1 & 1 & 0 & 211 \\
\hline 4,5 & 143 & 15 & 5 & 1 & 0 & 0 & 164 \\
\hline 5 & 151 & 15 & 17 & 0 & 0 & 2 & 185 \\
\hline Total & $\begin{array}{r}869 \\
85 \%\end{array}$ & 89 & 50 & $\begin{array}{l}4 \\
0,4 \%\end{array}$ & $\begin{array}{l}1 \\
0,1 \%\end{array}$ & $\begin{array}{l}5 \\
0,5 \%\end{array}$ & $\begin{array}{l}1018 \\
100 \%\end{array}$ \\
\hline
\end{tabular}

Tableau 3 : Accessibilité des SPND par tranches d'âge

Il est important de noter que le nombre de référents inférables ou nouveaux exprimés par des pronoms, disloqués ou non, est très faible, les trois degrés d'accessibilité les plus fréquents étant: mentionné sans compétiteurs, mentionné avec compétiteurs et enfin, physiquement présent, pour les SPD et SPND (voir graphiques 1 et 2).



- Mentionné, sans comp. Mentionné, avec comp. $\quad$ Mhysiquement présent

Graphique 1: Accessibilité des SPD par tranche d'âge 


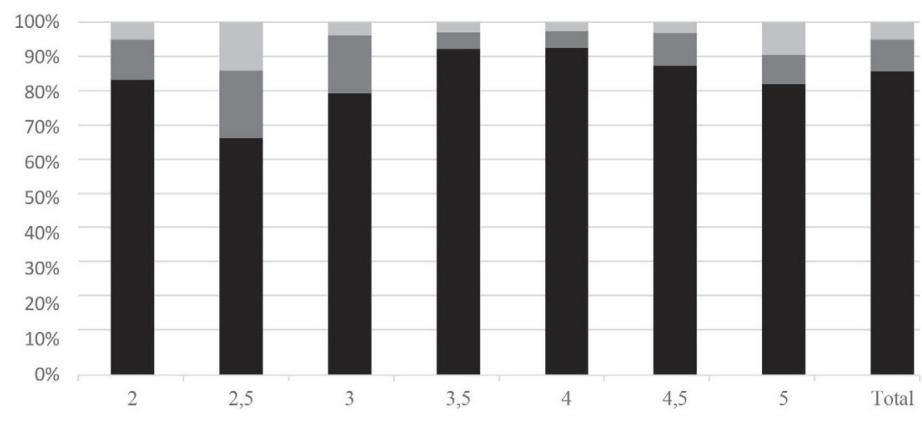

- Mentionné, sans comp. \entionné, avec comp. ॠ Physiquement présent

Graphique 2: Accessibilité des SPND par tranche d'âge

Pour les SPD et SNPD, les référents mentionnés sans compétiteurs sont les plus fréquents, et les référents mentionnés avec compétiteurs sont moins fréquents. Cependant, la proportion de SPD dont le référent est non-mentionnémais seulement physiquement présent est plus haute que celle des SPND ayant le même degré d'accessibilité. Les SPND semblent donc globalement avoir un degré d'accessibilité plus élevé que les SPD.

\subsubsection{Contraste}

Dans nos données, nous n'avons pas trouvé de cas clairs de SPND exprimant un topique contrastif. En comparaison, les SPD sont fréquemment utilisés dans des contextes contrastifs (52\% des occurrences, voir graphique 3 ).

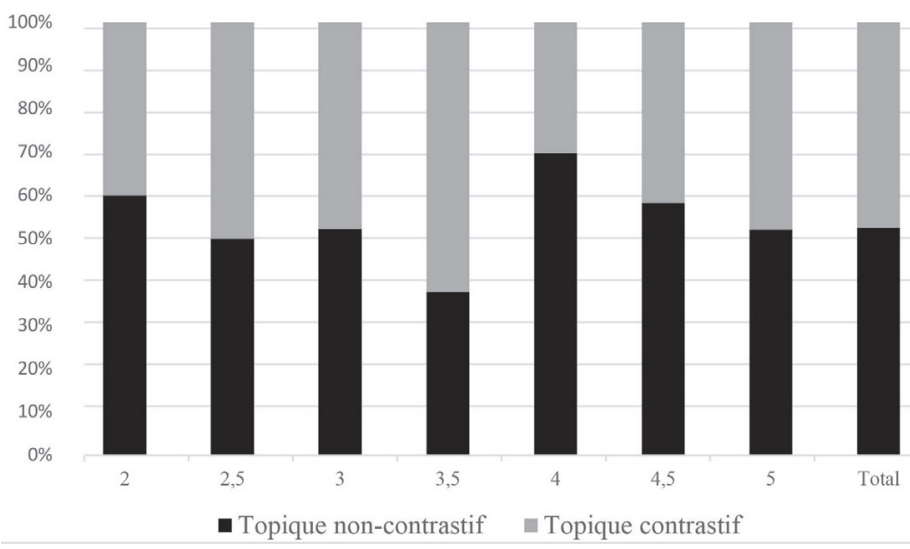

Graphique 3 : Proportion de topiques contrastifs et non-contrastifs 
Presque la moitié des SPD sont produits dans un contexte contrastif. Les pronoms démonstratifs (19) et personnels (20) peuvent être contrastifs.

19) ça c'est un monsieur, ça c'est Mickey, ça c'est quoi? (Hélö̈se, 2,5 ans)

20) Moi je fais un carré et toi tu mets au milieu dans le carré? (Mélanie, 3,5 ans)

Ces résultats montrent que les SPD et SPND ont des propriétés de SI distinctes. Chez les enfants de notre corpus, l'utilisation de la dislocation dans le cas des SP semble liée à l'accessibilité du sujet, ainsi qu'au contraste. Ainsi, les référents non-mentionnés dans le discours mais physiquement présents, ainsi que les topiques contrastifs seront plus souvent réalisés avec un SPD qu'avec un SPND. Par contre, tous les SP de notre corpus correspondent au topique de l'énoncé. Dans la section suivante, nous analysons les différences entre les SLD et les SLND.

\subsection{SLD et SLND}

Il est plus difficile de comparer les SL que les SP, à cause du très faible nombre de SLND (11 cas) et nous ne pouvons donc pas aisément identifier de différences au niveau quantitatif. Néanmoins, les SLND de notre corpus ont des propriétés que nous ne retrouvons pas dans nos SLD.

Conformément à De Cat $(2007 ; 2009)$ et Belletti et Manetti (2018), tous les SLD de notre corpus correspondent au topique de l'énoncé, comme illustré en (21). Dans cet exemple, le SLD le rhinocéros correspond au référent dont on parle, qui est l'animal dont les interlocuteurs sont en train de regarder l'image. L'enfant apporte une information nouvelle dans le discours, le fait qu'il ait des cornes, et l'attache au référent le rhinocéros.

21) Contexte: l'adulte et l'enfant regardent ensemble un livre d'images Alexandre: C'est un rhinocéros.

Adulte: Un rhinocéros?

Alexandre: Oui

Adulte: ça ressemble pas plutôt à un éléphant?

Alexandre: ben non regarde, il a ses cornes le n- le rhinocéros.

(Alexandre, 3 ans) 
En ce qui concerne les SLND, nous avons identifié un cas de sujet topique (22), ainsi qu'un cas produit dans une proposition faisant partie du cadre spatio-temporel de l'énoncé (23). Ainsi, dans l'exemple (22), le SLND Babouche correspond à l'entité dont on parle, et l'enfant apporte une information nouvelle à son sujet. Dans l'exemple (23), Lisa ne peut pas être défini comme le topique "dont on parle", appelé aboutness topic dans la littérature (Reinhart, 1981). Ce référent appartient à la proposition quand Lisa est là, qui contribue au cadre spatio-temporel pour lequel l'information véhiculée par la proposition principale est vraie. Ce type de cadre est parfois appelé topique scénique dans la littérature (Erteschik-Shir, 2007; Lahousse, 2007). Il est donc possible que l'enfant ne disloque que le topique d'à-propos de la phrase dans son ensemble. Une étude complémentaire sur les topiques d'à-propos et topiques scéniques serait nécessaire pour tester cette hypothèse, avec un corpus plus large.

22) Victoire: Babouche il dort.

Adulte: Il dort?

Victoire: $\mathrm{mm}$

Adulte: Il est avec qui?

Victoire: maman

Adulte: C'est la maman?

Victoire: $m m$ Babouche aime baba (baba semble être une mauvaise prononciation de Dora, un autre personnage du livre que l'adulte et l'enfant sont en train de regarder)

Adulte: mouais Dora. (Victoire, 2 ans)

23) Adulte: Tu as trois paires de train?

Gabriel: oui un un train que Lisa elle a. J'ai pas le droit de faire quand Lisa est là parce que il fait trop fort. (Gabriel, 4 ans)

Les autres SLND de notre corpus (9 cas) peuvent être interprétés comme appartenant à des énoncés tout-focus, c'est-à-dire que l'ensemble de la proposition transmet une information nouvelle. Par exemple, en (24), l'énoncé une vieille sonna à la porte ne contient pas de référent produit dans le contexte précédent.

24) Adulte: Alors «La Belle et la Bête»

Adeline: Dans le château il y avait une bête. Le roi il voulait épouser une fille mais il n'était pas d'accord. Une fois une vieille sonna à la porte. (Adeline, 5 ans) 
Ces neuf énoncés tout-focus sont utilisés dans un contexte narratif. Quatre de ces cas sont produits avec une autre particularité du discours narratif: une tentative d'utilisation du passé simple (25).

25) La mère se meta à le suivre. (Baptiste, 3,5 ans)

Pour conclure cette section, les SLD et SLND de notre corpus sont produits dans des contextes différents. Les SLND appartiennent majoritairement à des énoncés tout-focus, qui sont eux-mêmes restreints au contexte narratif. Les SLD sont utilisés dans des énoncés topique-commentaire, comme chez l'adulte. Nous n'avons pas trouvé de cas de sur-généralisation de l'utilisation de la dislocation à des SL non-topique chez les 21 enfants de notre corpus.

\section{Discussion}

Les fréquences des différents types de sujets chez l'enfant correspondent à celles des autres études sur l'acquisition L1 du français: pour le sujet, les enfants produisent majoritairement des SPND, les SPD et les SLD sont également fréquents, et les SLND sont extrêmement rares chez l'enfant. Ainsi, sur les 1399 sujets analysés dans cette étude, seuls $25(1,8 \%)$ ne contiennent pas de pronom clitique. Cependant, neuf de ces SLND ont des propriétés que nous n'avons pas retrouvées dans les autres sujets: l'appartenance au focus. Les SPD et les SPND sont également utilisés avec des propriétés distinctes, les SPND étant majoritairement produits avec des topiques non-contrastifs et très accessibles, tandis que les SPD peuvent exprimer un topique contrastif et être moins accessibles.

Le pronom clitique étant réalisé pour $98,2 \%$ des sujets produits par les enfants de notre corpus, il pourrait être interprété comme étant obligatoire dans la grammaire de l'enfant, ce qui serait en accord avec les théories selon lesquelles le pronom clitique serait traité par l'enfant comme un marqueur morphologique (Gotowski, 2015; Legendre et al., 2010). Bien que le très faible nombre de SLND ne nous permette pas d'établir de conclusion définitive, il semble pourtant que les SLND et les SLD apparaissent dans des contextes bien distincts. L'hypothèse $\mathrm{du}$ pronom clitique comme marqueur morphologique ne peut pas expliquer cette différence au niveau des propriétés de la SI. 
En outre, plusieurs points montrent que l'hypothèse de Parisse (2008), selon laquelle la dislocation est utilisée par l'enfant pour éviter de produire un sujet long, ne peut non plus, à elle seule, expliquer les cas de dislocation chez l'enfant. Le premier point est que les SPD sont plus fréquents que les SLD. Dans le cas d'un SP, le sujet est plus long lorsqu'il est disloqué que lorsqu'il ne l'est pas, et la longueur de la phrase (en dehors de la périphérie) reste la même. L'hypothèse de Parisse (2008) ne peut donc pas expliquer la production de SPD. De plus, les différences au niveau de la SI entre SD et SND suggèrent que dès 2 ans, les enfants ont associé la construction avec les fonctions de l'adulte.

Comment expliquer alors que les SLND soient si peu fréquents? Une première tentative d'explication peut venir des travaux de De Cat (2007) et Lambrecht (1994), qui montrent qu'en français parlé, les SLND ne sont possibles que dans des énoncés tout-focus. Ainsi, les énoncés tout-focus peuvent être produits de deux façons: par des clivées (Dufter, 2008; Karssenberg, 2018; Karssenberg \& Lahousse, 2018), ou par des SLND. Il est donc possible que les enfants préfèrent utiliser les clivées plutôt que les SLND pour ce type de configuration de la SI. Le fait ques les enfants de notre corpus produisent des SLND tout-focus uniquement dans des contextes narratifs nous conduit à faire l'hypothèse que cette préférence pourrait être liée au type de discours. Les enfants pourraient alors privilégier des constructions syntaxiques différentes, en fonction du statut informationnel du sujet et du type de discours. L'étude de Roubaud et Sabio (2018) sur les types de sujets produits par des enfants entre 4 et 6 ans dans des contextes narratifs a en effet porté sur des résultats assez différents des nôtres, puisque $11,39 \%$ des sujets produits par les enfants de leur corpus sont des SLND. En terme de SI, seule l'accessibilité a été analysée par les auteurs. Leur étude montre qu'il y a une plus grande proportion de SLND que de SLD exprimant un référent nouveau, mais que pour les deux types de sujets, la majorité des référents sont connus. Ainsi, une étude plus poussée, visant à étudier les différents types de sujets produits par l'enfant (clivées incluses), considérant leurs propriétés au niveau de la syntaxe et de la SI, dans différents types de discours permettrait de déterminer si les enfants choisissent effectivement, la façon de produire le sujet en fonction de son statut informationnel et si ce choix dépend du type de discours. 


\section{Conclusion}

A partir d'une analyse de corpus de 1399 sujets produits par 21 enfants entre 2 et 5 ans du corpus TCOF, nous avons tenté d'identifier les facteurs conduisant les enfants à produire des énoncés disloqués. Nous avons montré que, conformément à la littérature, les enfants de notre corpus produisent très peu de SLND. Cependant, les hypothèses selon lesquelles la dislocation est utilisée par l'enfant pour diminuer la charge cognitive causée par des énoncés avec un sujet lexical ou par le traitement du clitique comme un marqueur flexionnel ne peuvent expliquer tous les cas de dislocation chez l'enfant. Les énoncés disloqués chez l'enfant respectent les contraintes de la structure de l'information propres au langage de l'adulte. Compte-tenu de nos données, notre hypothèse pour expliquer le très faible nombre de SLND chez l'enfant est que les enfants produisent peu d'énoncés toutfocus, au moins en dehors des clivées. Il s'agit de la seule configuration au niveau de la SI pour laquelle les SLND sont possibles en français parlé selon De Cat (2007) et Lambrecht (1994). De futures recherches prenant en compte les clivées sujet chez l'enfant nous permettront de tester cette hypothèse.

\section{Bibliographie}

ABEILlÉ A., GODARD D. \& SABIO, F. (2008), Two types of NP preposing in French, Paper presented at the 15th international conference on HPSG, National Institute of Information and Communications Technology, Keihanna, Stanford. CSLI Publications http://csli-publications.stanford.edu/

ASHBY W. (1988), The syntax, pragmatics and sociolinguistics of leftand right-dislocations in French, Lingua 75, 203-229.

AUGER J. (1994), More evidence for agreement-marking in colloquial French, in Ashby W., Mithun M., Perissinotto G. \& Raposo E. (eds), Linguistic perspectives on the romance languages, Philadelphia, John Benjamins, 177-198.

BARNES B. K. (1985), Left Detachment in Spoken Standard French, Amsterdam, Benjamins Publishing Company.

BELLETTI A. \& MANETTI C. (2018), Topics and passives in Italianspeaking children and adults, Language Acquisition 26, 153-182. 
LES SUJETS DISLOQUÉS ET NON-DISLOQUÉS CHEZ L'ENFANT FRANÇAIS

BLASCO-DULBECCO M. (1999), Les dislocations en français contemporain: étude syntaxique, Paris, Honoré Champion Editeur.

BÜRING D. (2016), (Contrastive) topic, in C. Féry \& S. Ishihara (Eds.), Handbook of Information Structure, Oxford, Oxford University Press, 64-85.

CAMPION E. (1984), Left disloction in Montreal French, thèse de doctorat, University of Pennsylvania.

CINQUE G. (1983), «Topic» constructions in some European languages and "connectedness", in Ehlich K. \& van Riemsdijk $\mathrm{H}$. (eds), Connectedness in sentence text and discourse, Tilburg, KBU, 7-42.

CULBERTSON J. (2010), Convergent evidence for categorical change in French: from subject clitic to agreement marker, Language $86,85-132$.

DE CAT C. (2002), French Dislocation, thèse de doctorat, University of York, UK.

DE CAT C. (2004), Apparent non-nominative subjects in L1 French, in Prévost P. \& Paradis J. (eds), The Acquisition of French in Different Contexts, Amsterdam, John Benjamins, 60-115.

DE CAT C. (2005), French Subject Clitics are not Agreement Markers, Lingua 115, 1195-1219.

DE CAT C. (2007), French dislocation, interpretation, syntax, acquisition, Oxford, Oxford University Press.

DE CAT C. (2009), Experimental Evidence for Preschoolers' Mastery of «Topic», Language Acquisition 16, 224-239.

DELAIS-ROUSSARIE E., DOETJES J. \& SLEEMAN P. (2004), Dislocation, in de Swart H. \& Corblin F. (eds), Handbook of French Semantics, Stanford, CSLI Publications, 501-529.

DUFTER A. (2008), On explaining the rise of c'est-clefts in French, in Detges U. \& Waltereit R. (eds.), The paradox of grammatical change: Perspectives from Romance, Amsterdam, John Benjamins, 31-56.

ERTESCHIK-SHIR N. (2007), Information structure. The syntaxdiscourse interface, Oxford, Oxford University Press.

FERDINAND A. (1996), The development of functional categories the acquisition of the subject in French (Vol. 23), The Hague, Holland Academic Graphics.

FERDINAND A. (1997), The Development of Phrase Structure in Child French, Linguistics in the Netherlands, 85-96. 
GOTOWSKI M. (2015), Subject doubling in child french, University of Pennsylvania Working Papers in Linguistics 21.

HAMANN C., RIZZI L. \& FRAUENFELDER U. (1996), The acquisition of subject and object clitics in French, in Clahsen H. (ed.), Generative perspectives on language acquisition, Amsterdam, John Benjamins, 309-334.

HORVÁTH M. G. (2016), Analyse pragmatico-discursive des SN disloqués à droite du français parlé, Revue Romane 51(2), 244270.

HORVÁTH M. G. (2018), Le français parlé informel, Stratégies de topicalisation, Berlin, De Gruyter Mouton.

KARSSENBERG L. (2018), Non-prototypical clefts in French: A corpus analysis of French il y a clefts, Berlin, De Gruyter Mouton.

KARSSENBERG L. \& LAHOUSSE, K. (2018), The information structure of French il y a clefts and c'est clefts: A corpus-based analysis, Linguistics 56, 513-548.

LABELLE M. \& VALOIS D. (1996), The status of post-verbal subjects in French child language, Probus 8, 53-80.

LAGAE V. (2012), Le paradigme des marqueurs thématiques en français: essai de typologie, in Comes E. \& Miculescu S. (éds), La construction d'un paradigme: Actes du XVIIe séminaire de didactique universitaire, Constanta 2010, Cluj, Echinox, 53-73.

LAHOUSSE K. (2007), Implicit stage topics: a case study in French, Discours 1.

LAMBRECHT K. (1987), On the status of SVO sentences in French discourse, in Tomlin R. S. (ed.), Coherence and grounding in discourse, Amsterdam, Benjamins.

LAMBRECHT K. (1994), Information structure and sentence form: topic, focus, and the mental representation of discourse referents, Cambridge, Cambridge University Press.

LAMBRECHT K. (2001), Dislocation, in Haspelmath M. (ed.), Language typology and language universals: an international handbook, Berlin, De Gruyter, 1050-1078.

LEGENDRE G., CULBERTSON, J., BARRIËRE I., NAZZI T. \& GOYET L. (2010), Experimental and Empirical Evidence for the Status and Acquisition of Subject Clitices and Agreement Marking in Adult and Child Spoken French, in Torrens V., Escobar L., Gavarro A., \& Mangado J. G. (eds), Movement and Clitics, Newcastle, Cambridge Scholars Publishing, 333-360. 
LEGENDRE G., VAINIKKA A., HAGSTROM P. \& TODOROVA M. (2002), Partial constraint ordering in child French syntax, Language Acquisition 10, 189-227.

PARISSE C. (2008), Left-dislocated subjects: A construction typical of young French-speaking children?, in Guijarro Fuentes P., Pilar Larrañaga M., \& Clibbens J. (eds), First Language Acquisition of Morphology and Syntax: Perspectives across Languages and Learners, Amsterdam, John Benjamins Publishing Company, 13-30.

PIERCE A. (1992), Language acquisition and syntactic theory: a comparative analysis of French and English child grammars, Dordrecht, The Netherlands, Kluwer.

PRINCE E. F. (1981), Towards a taxonomy of given-new information, in Cole P. (ed.), Radical pragmatics, New York, Academic Press, 223-255.

REINHART T. (1981), Pragmatics and linguistics: an analysis of sentence topics, Philosophica 27, 53-94.

REPP S. (2010), Defining "contrast» as an information-structural notion in grammar, Lingua 120, 1333-1345.

ROUBAUD M.-N. \& SABIO F. (2018), Syntaxe et affiliation du lexique: les réalisations du sujet chez les jeunes enfants (4-6 ans), Pratiques 177-178.

TCOF: Traitement de Corpus Oraux en Français [Corpus] Retrieved 10 July 2018, from ORTOLANG (Open Resources and TOols for LANGuage) ATILF (2018) 